\title{
An involution proof of the Alladi-Gordon key identity for Schur's partition theorem
}

\author{
James J.Y. Zhao \\ Dongling School of Economics and Management \\ University of Science and Technology Beijing \\ Beijing 100083, P.R. China \\ Center for Applied Mathematics \\ Tianjin University \\ Tianjin 300072, P.R. China \\ zhaojy@ustb.edu.cn
}

Submitted: Oct 23, 2012; Accepted: Mar 18, 2013; Published: Mar 24, 2013

Mathematics Subject Classifications: 05A17, 05A19

\begin{abstract}
The Alladi-Gordon identity $\sum_{k=0}^{j}\left(q^{i-k+1} ; q\right)_{k}\left[\begin{array}{l}j \\ k\end{array}\right] q^{(i-k)(j-k)}=1$ plays an important role for the Alladi-Gordon generalization of Schur's partition theorem. By using Joichi-Stanton's insertion algorithm, we present an overpartition interpretation for the Alladi-Gordon key identity. Based on this interpretation, we further obtain a combinatorial proof of the Alladi-Gordon key identity by establishing an involution on the underlying set of overpartitions.
\end{abstract}

Keywords: the Alladi-Gordon key identity; Joichi-Stanton's insertion algorithm; Schur's celebrated partition theorem; overpartitions

\section{Introduction}

Let $\mathbb{N}$ be the set of nonnegative integers. Let

$$
(a)_{k}=(a ; q)_{k}= \begin{cases}(1-a)(1-a q) \cdots\left(1-a q^{k-1}\right), & \text { if } k>0 \\ 1, & \text { if } k=0\end{cases}
$$

denote the common notation of $q$-shifted factorials [14]. Given $j, k \in \mathbb{N}$, let

$$
\left[\begin{array}{l}
j \\
k
\end{array}\right]=\frac{(q ; q)_{j}}{(q ; q)_{k}(q ; q)_{j-k}}
$$


denote the Gaussian coefficients, which are also called the $q$-binomial coefficients, or the Gaussian polynomials [6]. The main objective of this paper is to give a combinatorial proof of the following identity:

$$
\sum_{k=0}^{j}\left(q^{i-k+1} ; q\right)_{k}\left[\begin{array}{l}
j \\
k
\end{array}\right] q^{(i-k)(j-k)}=1,
$$

which we call the Alladi-Gordon key identity, since it was first introduced by Alladi and Gordon [4] in an equivalent form for the study of some generalization of Schur's celebrated partition theorem of 1926.

Schur [18] proved that the number of partitions of $m$ into parts with minimal difference 3 and with no consecutive multiples of 3 is equal to the number of partitions of $m$ into distinct parts $\equiv 1,2(\bmod 3)$. This significant result is now known as Schur's celebrated partition theorem of 1926. There are many proofs, refinements, and generalizations of Schur's partition theorem, see $[5,7,12,15]$ and references therein.

From the viewpoint of generating functions, each partition theorem implies a corresponding $q$-identity. The Alladi-Gordon key identity (1) is essentially equivalent to the following q-identity [4, Lemma 2] corresponding to Alladi and Gordon's notable generalization of Schur's partition theorem,

$$
\sum_{0 \leqslant m \leqslant \min \{i, j\}} \frac{q^{T_{i+j-m}+T_{m}}}{(q)_{i-m}(q)_{j-m}(q)_{m}}=\frac{q^{T_{i}+T_{j}}}{(q)_{i}(q)_{j}}
$$

where $i$ and $j$ are given nonnegative integers and $T_{i}=i(i+1) / 2$ is the $i$-th triangle number.

The Alladi-Gordon key identity turned out to have many interesting applications in the theory of partitions. Alladi and Berkovich [3, Eq. (2.1)]) obtained a double bounded version of Schur's partition theorem by generalizing an equivalent form of (2). Alladi, Andrews and Gordon [2, Lemma 2] introduced a three parameter generalization of (2) and obtained a generalization of the Göllnitz theorem [15], a higher level extension of Schur's partition theorem. Alladi, Andrews and Berkovich [1, Eq. (1.7)] further obtained a remarkable four parameter extension of the identity (2), which implies a four parameter partition theorem and thereby extends the Göllnitz theorem.

Due to its significance, the Alladi-Gordon key identity certainly deserves to be further studied. Alladi and Gordon [4] gave two proofs of (2), one combinatorial and the other algebraic. Alladi, Andrews and Berkovich [1] also pointed out that (2) is a special case of $q$-Chu-Vandermonde summation formula. In this paper we will present an overpartition interpretation of the left-hand side of (1) and then give another combinatorial proof of the Alladi-Gordon key identity.

This paper is organized as follows. In Section 2 we will review Joichi-Stanton's insertion algorithm for partitions and then give an overpartition interpretation of the AlladiGordon key identity. In Section 3, based on this interpretation, we will give an involution proof of the Alladi-Gordon key identity. 


\section{An overpartition interpretation of the key identity}

The aim of this section is to give a combinatorial interpretation of the left-hand side of (1) in terms of overpartitions. This is achieved by applying Joichi-Stanton's insertion algorithm for partitions.

Let us first review some definitions and notations about partitions. Recall that a partition $\lambda$ of $n \in \mathbb{N}$ with $k$ parts is denoted by a vector $\lambda=\left(\lambda_{1}, \lambda_{2}, \ldots, \lambda_{k}\right)$, where $\lambda_{1} \geqslant \lambda_{2} \geqslant \cdots \geqslant \lambda_{k} \geqslant 0$ and $\sum_{i=1}^{k} \lambda_{i}=n$. The number $n$ is called the size of $\lambda$, denoted by $|\lambda|$. For convenience, the length of $\lambda$ is defined to be the number $k$ of nonnegative parts of $\lambda$, denoted by $\ell(\lambda)$. (Note that $\ell(\lambda)$ usually enumerates the number of positive parts.) An overpartition is a partition in which the first occurrence of a number may be overlined. For example, $\lambda=(9, \overline{7}, 6,5,5, \overline{2}, 2, \overline{1})$ is an overpartition with three overlined parts. An ordinary partition can also be treated as an overpartition with no overlined parts. The concept of overpartition was first proposed by Corteel and Lovejoy [11] while studying basic hypergeometric series. For deeper research on overpartitons, see for instance $[8,9$, $13,17]$.

An overpartition can also be understood as a pair of partitions $(\alpha, \beta)$, where $\alpha$ is a partition with distinct parts and $\beta$ is an ordinary partition. Joichi and Stanton [16] found the following fundamental bijection which can be restated in terms of overpartitions.

Theorem 1. There is a one-to-one correspondence between overpartitions with $n$ nonnegative parts, and pairs of partitions $(\alpha, \beta)$, where $\alpha$ is a partition with distinct parts from the set $\{0,1,2, \ldots, n-1\}$ and $\beta$ is a partition with $n$ nonnegative parts.

The above correspondence can be described as an insertion algorithm [16, Algorithm $\Phi]$. Given an ordinary partition $\beta$, we may insert a part $m$ into $\beta$, by adding 1 to the first $m$ parts of $\beta$, and putting an overline above the $(m+1)$-th part. Moreover, we can add other distinct parts in the same way.

Example 2. If $\alpha=(5,3,0)$ and $\beta=(9,6,5,2,2,0)$, then we get an overpartition $(\overline{11}, 8,7, \overline{3}, 3, \overline{0})$.

To give a combinatorial interpretation of (1), we shall assign a weight to each overlined part of an overpartition. As in [10], each overlined part of an overpartition has the same weight. For example, $\lambda=(9, \overline{7}, 6,5,5, \overline{2}, 2, \overline{1}, 0)$ with a weight 3 endowed in each overline is displayed in Figure 1, where each overline of $\lambda$ is represented by a row of three hollow dots, and the part zero is represented by the symbol $\varnothing$.

Given $0 \leqslant k \leqslant j \leqslant i$, let $A(i, k)$ denote the set of partitions into distinct parts from the set $\{i-k+1, i-k+2, \ldots, i\}$ plus the empty partition, and let $B(j, k)$ denote the set of partitions into $k$ nonnegative parts with each part not exceeding $j-k$. It is easy to see

$$
\sum_{\lambda \in A(i, k)}(-1)^{\ell(\lambda)} q^{|\lambda|}=\left(q^{i-k+1} ; q\right)_{k}
$$




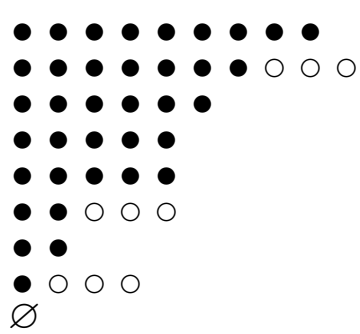

Figure 1: An overpartition $\lambda=(9, \overline{7}, 6,5,5, \overline{2}, 2, \overline{1}, 0)$ with a weight 3 in each overline.

and it is well known [6, Theorem 3.1] that

$$
\sum_{\lambda \in B(j, k)} q^{|\lambda|}=\left[\begin{array}{l}
j \\
k
\end{array}\right]
$$

We now come to the main result of this section. For a weighted overpartition $\lambda$, let $o l(\lambda)$ denote the number of overlined parts of $\lambda$, and let $w(\lambda)$ denote the weight assigned to each overlined part of $\lambda$. Given $0 \leqslant k \leqslant j \leqslant i$, let $O(i, j, k)$ denote the set of weighted overpartitions into $k$ nonnegative parts, which satisfy the following three conditions:

(1) each part is less than or equal to $j-1$;

(2) for $k \geqslant 2$ and each $1 \leqslant s \leqslant k-1$ there are at least $k-s$ overlined parts to the right of $j-s$ if it occurs as a part;

(3) and, each overline is endowed with a weight $i-k+1$.

Note that the empty partition is the sole element of $O(i, j, 0)$. For fixed $i, j$ satisfying $0 \leqslant j \leqslant i$, let

$$
O(i, j)=\biguplus_{k=0}^{j} O(i, j, k)
$$

The main result of this section is as follows.

Theorem 3. Given $0 \leqslant j \leqslant i$, we have

$$
\sum_{k=0}^{j}\left(q^{i-k+1} ; q\right)_{k}\left[\begin{array}{l}
j \\
k
\end{array}\right] q^{(i-k)(j-k)}=\sum_{\lambda \in O(i, j)}(-1)^{o l(\lambda)} q^{|\lambda|+o l(\lambda) w(\lambda)} q^{(i-\ell(\lambda))(j-\ell(\lambda))} .
$$

Proof. By (5), it suffices to prove that

$$
\left(q^{i-k+1} ; q\right)_{k}\left[\begin{array}{l}
j \\
k
\end{array}\right]=\sum_{\lambda \in O(i, j, k)}(-1)^{o l(\lambda)} q^{|\lambda|+o l(\lambda)(i-k+1)},
$$

since for each $\lambda \in O(i, j, k)$ we have $\ell(\lambda)=k$ and $w(\lambda)=i-k+1$. It is clearly true for $k=0$. In the following we may assume that $k \geqslant 1$. In view of (3) and (4), we only 
need to give a weight-preserving bijection $\bar{\Phi}$ between the set $A(i, k) \times B(j, k)$ and the set $O(i, j, k)$. Actually, we can obtain $\bar{\Phi}$ by using Joichi-Stanton's insertion algorithm.

For any given pair $(\gamma, \beta) \in A(i, k) \times B(j, k)$, define $\bar{\Phi}(\gamma, \beta)$ to be the partition $\lambda$ obtained as follows.

(i) If $\gamma$ is the empty partition, then let $\lambda=\beta$. By Property (2) of the definition of $O(i, j, k)$, it is clear that $B(j, k) \subseteq O(i, j, k)$. Therefore, in this case we have $\lambda \in O(i, j, k)$.

(ii) If $\gamma$ is not the empty partition, then let $\bar{\gamma}$ denote the partition obtained from $\gamma$ by decreasing each part by $i-k+1$. Therefore, $\bar{\gamma}$ is a partition into distinct parts from the set $\{0,1, \ldots, k-1\}$. Now we insert $\bar{\gamma}$ into $\beta$ by applying Joichi-Stanton's insertion algorithm, and obtain an overpartition $\lambda$ with at least one overlined part. If each overline is endowed with a weight $i-k+1$, then it is routine to verify that the weighted overpartition $\lambda$ lies in $O(i, j, k)$. Note that the number of parts of $\gamma$ is equal to the number of overlined parts of $\lambda$. Thus

$$
(-1)^{\ell(\gamma)} q^{|\gamma|} q^{|\beta|}=(-1)^{o l(\lambda)} q^{|\lambda|+o l(\lambda)(i-k+1)} .
$$

It remains to show that $\bar{\Phi}$ is reversible. There are two cases to consider.

(i') If $\lambda \in O(i, j, k)$ and there are no overlined parts in $\lambda$, then again by Property (2) of the definition of $O(i, j, k)$, we must have $\lambda \in B(j, k)$. In this case, let $\bar{\Phi}^{-1}(\lambda)=$ $(\gamma, \beta)$, where $\gamma$ is the empty partition and $\beta=\lambda$.

(ii') If $\lambda \in O(i, j, k)$ and there is at least one overlined part in $\lambda$, then by reversing the insertion algorithm, we will obtain a pair of partitions $(\bar{\gamma}, \beta)$. Clearly, $\bar{\gamma}$ is a partition into distinct parts from the set $\{0,1, \ldots, k-1\}$ since there are $k$ parts in $\lambda$. It is also clear that $\beta$ has only $k$ parts. We further need to show that each part of $\beta$ is not exceeding $j-k$. Suppose that there are $t$ overlined parts to the right of $\lambda_{1}$, then $\beta_{1}=\lambda_{1}-t$. Assume that $\lambda_{1}=j-s$ for some $1 \leqslant s \leqslant k-1$. By Property (2) of the definition of $O(i, j, k)$ we have $t \geqslant k-s$. Therefore, $\beta_{1}=\lambda_{1}-t=j-s-t \leqslant j-k$.

This completes the proof.

The following example gives an illustration of the map $\bar{\Phi}$ of the above proof.

Example 4. For $i=9, j=6, k=3, \gamma=(8,7) \in A(9,3), \beta=(3,3,2) \in B(6,3)$. We shall transform $(\gamma, \beta)$ into $\lambda \in O(9,6,3)$ in two steps.

(1) Change $\gamma=(8,7)$ into $\bar{\gamma}=(1,0)$ by decreasing each part by 7 .

(2) Insert $\bar{\gamma}=(1,0)$ into $\beta=(3,3,2)$ to obtain an overpartition $\lambda=(\overline{4}, \overline{3}, 2) \in O(9,6,3)$, where each overline contains a weight 7 . See Figure 2.

By reversing the procedure it is easy to obtain $(\gamma, \beta)$ from $\lambda$. 


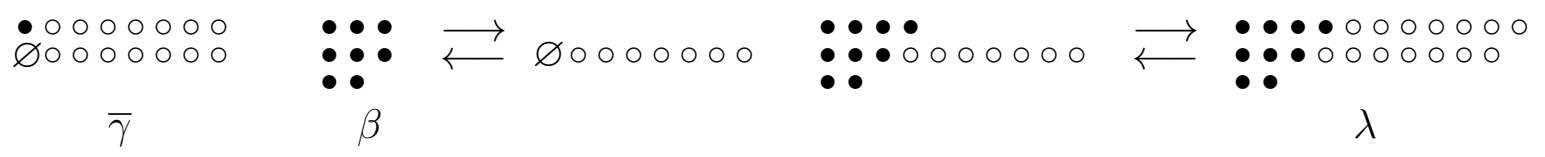

Figure 2: Insertion of $\bar{\gamma}=(1,0)$ into $\beta=(3,3,2)$ leads to $\lambda=(\overline{4}, \overline{3}, 2)$, where $\bar{\gamma}$ is represented as the overpartition $(\overline{1}, \overline{0})$ and each overline has a weight 7 endowed.

\section{Combinatorial proof of the Alladi-Gordon key iden- tity}

The aim of this section is to prove the following result by constructing an involution on the set $O(i, j)$.

Theorem 5. Given $0 \leqslant j \leqslant i$, we have

$$
\sum_{\lambda \in O(i, j)}(-1)^{o l(\lambda)} q^{|\lambda|+o l(\lambda) w(\lambda)} q^{(i-\ell(\lambda))(j-\ell(\lambda))}=1,
$$

where $O(i, j)$ is as defined in (5).

Combining Theorem 3, this provides a combinatorial proof of the the Alladi-Gordon key identity.

To prove Theorem 5, we first give a decomposition of $O(i, j)$. For $\lambda \in O(i, j)$ let $\lambda_{t}$ denote the largest overlined part of $\lambda$. Let

$$
\begin{aligned}
& O_{1}(i, j)=\left\{\lambda \in O(i, j) \mid \text { ol }(\lambda) \geqslant 1, \lambda_{t}=j-\ell(\lambda)+o l(\lambda)-1\right\}, \\
& O_{2}(i, j)=\left\{\lambda \in O(i, j) \mid \text { ol }(\lambda) \geqslant 1, \lambda_{t}<j-\ell(\lambda)+o l(\lambda)-1\right\}, \\
& O_{3}(i, j)=\{\lambda \in O(i, j) \mid \text { ol }(\lambda)=0\} .
\end{aligned}
$$

For the convenience, let the empty partition belong to $O_{3}(i, j)$.

Lemma 6. For $0 \leqslant j \leqslant i$, we have

$$
O(i, j)=O_{1}(i, j) \uplus O_{2}(i, j) \uplus O_{3}(i, j) .
$$

Proof. It is clear that $O_{1}(i, j), O_{2}(i, j)$ and $O_{3}(i, j)$ are disjoint from each other. It suffices to show that for each $\lambda \in O(i, j)$ with $o l(\lambda) \geqslant 1$, we have $\lambda_{t} \leqslant j-\ell(\lambda)+o l(\lambda)-1$. Otherwise, suppose that $\lambda_{t}=j-s$ and $s<\ell(\lambda)-o l(\lambda)+1$. By Property (2) of the definition of $O(i, j, k)$, there are at least $\ell(\lambda)-s \geqslant o l(\lambda)$ overlined parts to the right of $\lambda_{t}$, contradicting the definition of $o l(\lambda)$. This completes the proof. 5 .

With the above decomposition of $O(i, j)$, we can now give a bijective proof of Theorem 
Proof of Theorem 5. For $\lambda \in O(i, j)$, let

$$
f(\lambda)=(-1)^{o l(\lambda)} q^{|\lambda|+o l(\lambda) w(\lambda)} q^{(i-\ell(\lambda))(j-\ell(\lambda))} .
$$

To give a bijective proof, we define an involution, denoted $\Psi$, acting on $O(i, j)$ as follows:

(1) If $\lambda \in O_{1}(i, j)$, then let $\Psi(\lambda)$ denote the overpartition obtained from $\lambda$ by removing the largest overlined part $\lambda_{t}$. In this case, we have

$$
\begin{aligned}
\ell(\Psi(\lambda)) & =\ell(\lambda)-1 \\
o l(\Psi(\lambda)) & =o l(\lambda)-1 \\
|\Psi(\lambda)| & =|\lambda|-\lambda_{t} \\
& =|\lambda|-(j-\ell(\lambda)+o l(\lambda)-1) .
\end{aligned}
$$

By Property 3 of the definition of $O(i, j, k)$, we have

$$
w(\Psi(\lambda))=i-\ell(\Psi(\lambda))+1=(i-\ell(\lambda)+1)+1=w(\lambda)+1 .
$$

It is routine to verify that $f(\Psi(\lambda))+f(\lambda)=0$. Note that if $o l(\lambda)=1$, then clearly $\Psi(\lambda) \in O_{3}(i, j)$. If $o l(\lambda)>1$, then we must have $\Psi(\lambda) \in O_{2}(i, j)$ since

$$
\Psi(\lambda)_{t}<\lambda_{t}=j-\ell(\lambda)+o l(\lambda)-1=j-\ell(\Psi(\lambda))+o l(\Psi(\lambda))-1
$$

(The inequality $\Psi(\lambda)_{t}<\lambda_{t}$ follows from the definition of overpartitions.) In both cases, we have $\ell(\Psi(\lambda))=\ell(\lambda)-1 \leqslant j-1$.

(2) If $\lambda \in O_{2}(i, j)$, then we must have $j>\ell(\lambda)$. Otherwise, if $j=\ell(\lambda)$, then $\lambda_{t}<$ $o l(\lambda)-1$, contradicting the fact that the overlined parts of an overpartition are distinct nonnegative integers. Then let $\Psi(\lambda)$ denote the overpartition obtained from $\lambda$ by inserting an overlined part $j-\ell(\lambda)+o l(\lambda)-1$. Clearly, $\Psi(\lambda) \in O_{1}(i, j)$ and $\Psi(\Psi(\lambda))=\lambda$.

(3) If $\lambda \in O_{3}(i, j)$, then we define $\Psi(\lambda)$ as follows according to whether $j>\ell(\lambda)$. If $j>\ell(\lambda)$, then let $\Psi(\lambda)$ denote the overpartition obtained from $\lambda$ by inserting an overlined part $j-\ell(\lambda)-1$. In this case, it is clear that $\Psi(\lambda) \in O_{1}(i, j)$ and $\Psi(\Psi(\lambda))=\lambda$. If $j=\ell(\lambda)$, then $\lambda$ must be the partition $(\underbrace{0,0, \ldots, 0}_{j^{\prime} s})$. Otherwise, we will have $\lambda_{1}>0$, and by Property 2 of $O(i, j, j)$ there must be at least one overlined part in $\lambda$ contradicting $o l(\lambda)=0$. In this case let $\Psi(\lambda)=\lambda$.

By the involution $\Psi$ of $O(i, j)$, we have

$$
\sum_{\lambda \in O(i, j)} f(\lambda)=\sum_{\lambda=(\underbrace{0,0, \ldots, 0}_{j^{\prime} s})} f(\lambda)=1 .
$$

This completes the proof. 
In fact, there is a graphical representation of the involution $\Psi$ of $O(i, j)$ in the above proof, which seems more convenient and intuitive. Since each $\lambda \in O(i, j)$ contributes a term

$$
f(\lambda)=(-1)^{o l(\lambda)} q^{|\lambda|+o l(\lambda) w(\lambda)} q^{(i-\ell(\lambda))(j-\ell(\lambda))},
$$

we may consider $\lambda$ as a pair of partitions $(\lambda, \widehat{\lambda})$, where $\widehat{\lambda}$ is the unique rectangular partition $(\underbrace{i-\ell(\lambda), \ldots, i-\ell(\lambda)}_{(j-\ell(\lambda))^{\prime} s})$.

Example 7. Take $i=9, j=6$ and let $\lambda=(\overline{4}, \overline{3}, 2)$. In this case, we have $\widehat{\lambda}=(6,6,6)$, $o l(\lambda)=2, \ell(\lambda)=3, w(\lambda)=i-\ell(\lambda)+1=7$, and hence $\lambda \in O_{1}(i, j)$. Thus, $\Psi(\lambda)=$ $(\overline{3}, 2) \in O_{2}(i, j)$ and $\widehat{\Psi(\lambda)}=(7,7,7,7)$. Geometrically, $\Psi$ acts on $\lambda$ (or equivalently $\left.(\lambda, \widehat{\lambda})\right)$ as illustrated in Figure 3: remove a row of dots representing the largest overlined part, add a hollow dot to the rightmost of each overlined part, and append a hook to the top-left of the diagram of $\widehat{\lambda}$.

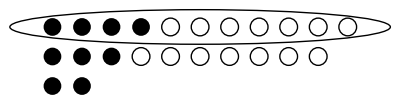

$\lambda$

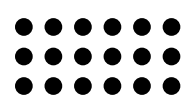

$\widehat{\lambda}$

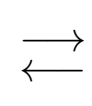

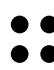

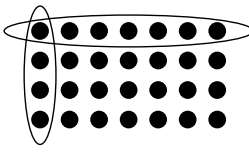

$\widehat{\Psi(\lambda)}$

Figure 3: The involution $\Psi$.

\section{Acknowledgements}

I am grateful to Arthur L.B. Yang, Qing-Hu Hou, and Guoce Xin for valuable comments leading to an improvement of an earlier version. I wish to thank the anonymous referee for a very careful reading and for helpful suggestions. This work was supported by the National Natural Science Foundation of China Grant No.71171018, Program for New Century Excellent Talents in University, and the Fundamental Research Funds for the Central Universities.

\section{References}

[1] K. Alladi, G.E. Andrews, and A. Berkovich. A new four parameter $q$-series identity and its partition implications. Invent. Math., 153(2):231-260, 2003.

[2] K. Alladi, G.E. Andrews, and B. Gordon. Generalizations and refinements of a partition theorem of Göllnitz. J. Reine Angew. Math., 460:165-188, 1995.

[3] K. Alladi and A. Berkovich. A double bounded version of Schur's partition theorem. Combinatorica, 22(2):151-168, 2002. 
[4] K. Alladi and B. Gordon. Generalizations of Schur's partition theorem. Manuscr. Math., 79:113-126, 1993.

[5] K. Alladi and B. Gordon. Schur's partition theorem, companions, refinements and generalizations. Trans. Amer. Math. Soc., 347(5):1591-1608, 1995.

[6] G.E. Andrews. The Theory of Partitions. Encyclopedia of Mathematics and its Applications, vol. 2, Rota, G.-C. (ed.) Addison-Wesley, Reading, 1976. Reissued: Cambridge University Press, Cambridge, 1998.

[7] D.M. Bressoud. A combinatorial proof of Schur's 1926 partition theorem. Proc. Amer. Math. Soc., 79(2):338-340, 1980.

[8] K. Bringmann and J. Lovejoy. Overpartitions and class numbers of binary quadratic forms. Proc. Natl. Acad. Sci. U.S.A., 106(14):5513-5516, 2009.

[9] W.Y.C. Chen, D.D.M. Sang, and D.Y.H. Shi. The Rogers-Ramanujan-Gordon theorem for overpartitions. Proc. London Math. Soc., to appear.

[10] W.Y.C. Chen and J.J.Y. Zhao. The Gaussian coefficients and overpartitions. Discrete Math., 305(1-3):350-353, 2005.

[11] S. Corteel and J. Lovejoy. Overpartitions. Trans. Amer. Math. Soc., 356(4):16231635, 2004.

[12] S. Corteel and J. Lovejoy. An iterative-bijective approach to generalizations of Schur's theorem. Europ. J. Combin., 27(4):496-512, 2006.

[13] J.-F. Fortin, P. Jacob, and P. Mathieu. Generating function for K-restricted jagged partitions. Electron. J. Combin., 12:R12, 2005.

[14] G. Gasper and M. Rahman. Basic Hypergeometric Series, Second Edition. Encyclopedia of Mathematics and its Applications, vol. 96. Cambridge University Press, Cambridge, 2004.

[15] H. Göllnitz. Partitionen mit Differenzenbedingungen. J. Reine Angew. Math., 225:154-190, 1967.

[16] J.T. Joichi and D. Stanton. Bijective proof of basic hypergeometric series identities. Pacific J. Math., 127(1):103-120, 1987.

[17] J. Lovejoy and O. Mallet. Overpartition pairs and two classes of basic hypergeometric series. Adv. Math., 217(1):386-418, 2008.

[18] I.J. Schur. Zur Additiven Zahlentheorie. S.-B. Preuss. Akad. Wiss. Phys.-Math. Kl., 1926, pp. 488-495. (Reprinted in I. Schur, Gesammelte Abhandlungen, vol. 2, Springer Verlag, Berlin, 1973, pp. 43-50.) 